\title{
MPGES-1-derived PGE2 suppresses CD80 expression on tumor- associated phagocytes to inhibit anti-tumor immune responses in breast cancer
}

\author{
Catherine Olesch ${ }^{1, *}$, Weixiao Sha ${ }^{1, *}$, Carlo Angioni ${ }^{2}$, Lisa Katharina Sha ${ }^{1}$, Elias Açaf ${ }^{1}$, \\ Paola Patrignani ${ }^{3}$, Per-Johan Jakobsson ${ }^{4}$, Heinfried H. Radeke ${ }^{5}$, Sabine Grösch ${ }^{2}$, \\ Gerd Geisslinger ${ }^{2}$, Andreas von Knethen ${ }^{1}$, Andreas Weigert ${ }^{1}$ and Bernhard Brüne ${ }^{1}$ \\ ${ }^{1}$ Institute of Biochemistry I, Faculty of Medicine, Goethe-University Frankfurt, Frankfurt, Germany \\ 2 Institute of Clinical Pharmacology/ZAFES, Faculty of Medicine, Goethe-University Frankfurt, Frankfurt, Germany \\ 3 Department of Neuroscience, Imaging and Clinical Sciences and Center of Excellence on Aging (CeSI), "G. d'Annunzio" \\ University, Chieti, Italy \\ ${ }^{4}$ Department of Medicine, Rheumatology Research Unit, Karolinska Institutet, Stockholm, Sweden \\ ${ }^{5}$ Pharmazentrum Frankfurt/ZAFES, Faculty of Medicine, Goethe-University Frankfurt, Frankfurt, Germany \\ * These authors contributed equally to the work
}

Correspondence to: Bernhard Brüne, email: b.bruene@biochem.uni-frankfurt.de

Andreas Weigert, email: weigert@biochem.uni-frankfurt.de

Keywords: prostaglandins, microenvironment, macrophage polarization, costimulation, cytotoxicity

Received: July 08, $2014 \quad$ Accepted: February 13, $2015 \quad$ Published: March 14, 2015

This is an open-access article distributed under the terms of the Creative Commons Attribution License, which permits unrestricted use, distribution, and reproduction in any medium, provided the original author and source are credited.

\section{ABSTRACT}

Prostaglandin $\mathrm{E}_{2}\left(\mathrm{PGE}_{2}\right)$ favors multiple aspects of tumor development and immune evasion. Therefore, microsomal prostaglandin E synthase (mPGES-1/-2), is a potential target for cancer therapy. We explored whether inhibiting mPGES-1 in human and mouse models of breast cancer affects tumor-associated immunity. A new model of breast tumor spheroid killing by human PBMCs was developed. In this model, tumor killing required CD80 expression by tumor-associated phagocytes to trigger cytotoxic $T$ cell activation. Pharmacological mPGES-1 inhibition increased CD80 expression, whereas addition of $\mathrm{PGE}_{2}$, a prostaglandin $\mathrm{E} 2$ receptor 2 (EP2) agonist, or activation of signaling downstream of EP2 reduced CD80 expression. Genetic ablation of mPGES-1 resulted in markedly reduced tumor growth in PyMT mice. Macrophages of mPGES-1/- PyMT mice indeed expressed elevated levels of CD80 compared to their wildtype counterparts. CD80 expression in tumor-spheroid infiltrating $\mathrm{mPGES}-1^{-/-}$macrophages translated into antigen-specific cytotoxic $\mathrm{T}$ cell activation. In conclusion, MPGES-1 inhibition elevates CD80 expression by tumorassociated phagocytes to restrict tumor growth. We propose that mPGES-1 inhibition in combination with immune cell activation might be part of a therapeutic strategy to overcome the immunosuppressive tumor microenvironment.

\section{INTRODUCTION}

Tumor-associated inflammation is known to drive carcinogenesis and thus, appears as a rational pharmacological target. In line, new chemotherapy concepts aim at de novo triggering and/or restoring immunological responses against tumors $[1,2]$. In addition, activation of tumor-infiltrating phagocytes by toll-like receptor (TLR) agonists emerged as a promising therapeutic option $[2,3]$. Phagocyte activation not only promotes tumor-associated antigen presentation and upregulates costimulatory B7 family molecules such as $\mathrm{CD} 80 / \mathrm{CD} 86[4,5]$ to trigger anti-tumor lymphocyte activation, but also initiates counter-regulatory signals that attenuate activation. Counter-regulatory mediators that are part of the activation-induced inflammatory process unfortunately drive carcinogenesis through various pathways [4, 6, 7]. This process is often 
overlooked during immunotherapy, since therapyinflicted inflammation is primarily considered as a sign of successful immune activation. One mediator of tumorpromoting inflammation is prostaglandin E2 $\left(\mathrm{PGE}_{2}\right)$. $\mathrm{PGE}_{2}$ is produced by cyclooxygenases $(\mathrm{COX}-1 /-2)$ and downstream cytosolic or microsomal prostaglandin synthases (cPGES, mPGES-1/-2) [8]. Under inflammatory conditions, the enzymes cyclooxygenase-2 (COX-2) and microsomal prostaglandin E synthase-1 (mPGES-1) are rapidly induced and functionally couple to accomplish $\mathrm{PGE}_{2}$ synthesis $[9,10]$. Inhibition of COX-2 with the intention to block $\mathrm{PGE}_{2}$ formation is widely used to limit inflammation and pain and has lately been proposed for treating or preventing cancer. However, COX-2 inhibition affects other prostanoids besides $\mathrm{PGE}_{2}$. This prompted the development of mPGES-1 inhibitors to selectively target $\mathrm{PGE}_{2}$ [11]. The role of $\mathrm{COX}$-2-derived $\mathrm{PGE}_{2}$ in the pathogenesis of cancer is well characterized. It acts as a tumor cell survival/proliferation factor, promotes angiogenesis, and modulates immune responses $[12,13]$. Regarding immune regulation, $\mathrm{PGE}_{2}$ favors the generation of human and murine myeloid-derived suppressor cells (MDSC), inhibits cytotoxic T cells (CTLs), and suppresses phagocyte activation and/or maturation under inflammatory conditions [8, 14-16]. However, only limited information is available whether and how $\mathrm{PGE}_{2}$ regulates the crosstalk of phagocytes and $T$ cells in the tumor microenvironment. In addition, it is unclear whether mPGES-1 inhibition fully recapitulates effects of COX-2 inhibition, which so far is widely used to investigate the impact of $\mathrm{PGE}_{2}$ on immune cell function.

To address these questions, we used a direct human PBMC - 3D tumor spheroid co-culture system to mimic human breast tumor development [17]. First, we explored whether this experimental set up can be used to monitor immune cell - tumor interactions and defined conditions that are required to mount an anti-tumoral response. As markers of such an immune response we analyzed shrinkage of tumor spheroids, CD80/CD86 expression on antigen-presenting cells (APCs), and granzyme B (GrB) expression by CTLs $[18,19]$. These markers were altered upon modulating $\mathrm{PGE}_{2}$ production and signalling. Second, we investigated the impact of mPGES-1 during breast cancer development in mice expressing the polyoma middle T oncogene (PyMT) under the control of the mouse mammary tumor virus (MMTV) promoter, which induces spontaneous mammary tumors [20]. Reduced growth of mPGES-1/- tumors was correlated to altered phagocyte activation, which we linked to CTL activation in vitro. Finally, we discuss potential therapeutic implications that arise from our data.

\section{RESULTS}

\section{CD80 expression on phagocytes induces GrBhi CTLs and tumor killing}

We compared the impact of $\mathrm{COX}-2$ versus mPGES-1 inhibition on human experimental tumorassociated immunity in an authentic in vitro tumor model composed of human MCF-7 breast cancer cells and human PBMCs. Culturing human PBMCs from healthy donors with MCF-7 breast cancer tumor spheroids was expected to result in spontaneous allogeneic responses [26]. Surprisingly, this was not the case. PBMCs required addition of lipopolysaccharide (LPS) and interferon- $\gamma$ (IFN- $\gamma$ ) or anti-CD3/anti-CD28 beads, i.e. immune cell activation, to reduce tumor spheroid size (Figure 1A,B). Challenging tumor spheroids with LPS in the absence of PBMCs did not affect spheroid sizes (data not shown). Addition of IFN- $\gamma$ augmented the LPS-induced anti-tumoral activity of PBMCs, followed by spheroid size reduction (Figure 1A,B). Stimulation with antiCD3/anti-CD28 beads, resulting among others in high IFN- $\gamma$ production, was equally effective (Figure 1A,B). These data suggest that human PBMCs require a strong activating stimulus to overcome the suppressive tumor microenvironment. The tumor microenvironment was suppressive even in our MHC mismatched setting and in the presence of danger-associated molecular patterns derived from necrotic cells in the spheroid core [21]. Next, we explored markers of immune cell activation that could be used to monitor an efficient immune response against tumor spheroids. LPS increased the number of GrB-expressing (GrB ${ }^{\text {hi }}$ ) CTLs (Figure 1C, Figure S1A). This was further increased by IFN- $\gamma$ (Figure 1C). Thus, a cytotoxic $\mathrm{T}$ cell response correlated with PBMCmediated reduction of tumor spheroid size. Next, we investigated the phagocyte phenotype that was required for CTL activation. LPS-activated CD14 ${ }^{+}$CD $11 \mathrm{c}^{+}$ human phagocytes expressed significantly higher levels of the inflammatory macrophage/DC marker CD80 in tumor spheroids, which again further increased upon addition of IFN- $\gamma$ (Figure 1D, Figure S1B). In contrast, CD86 expression was not significantly altered after LPS or LPS/IFN- $\gamma$ challenge (Figure 1E). Expression of CD206, a marker of anti-inflammatory macrophages, was significantly reduced upon LPS stimulation (Figure 1F). Next, we asked whether CD80 expression on phagocytes was a prerequisite for CTL activation and tumor spheroid size reduction. Therefore, CD80 on spheroid-infiltrating phagocytes was blocked using an anti-CD80 antibody. This treatment indeed reduced the numbers of $\mathrm{GrB}^{\text {hi }}$ CTLs and blunted tumor spheroid killing (Figure 2A,B). Neither a CD86-interfering antibody nor the isotype control affected numbers of $\mathrm{GrB}^{\text {hi }}$ CTLs or tumor spheroid killing 
(Figure 2A,B).

\section{Activation of PBMCs induces COX-2/mPGES-1- derived PGE2 in MCF-7 spheroid cocultures}

We asked whether $\mathrm{PGE}_{2}$ affects immune cell activation in our in vitro tumor killing model. Importantly, although 3D assembly of cancer cells can trigger expression of the $\mathrm{PGE}_{2}$ producing machinery, e.g. COX2 [21], MCF-7 spheroids neither upregulated COX-2 mRNA nor produced PGE (Figure S2A,B). When PBMCs were cocultured with MCF-7 breast cancer spheroids, production of relevant $\mathrm{PGE}_{2}$ amounts was absent (Figure 3A). However, activation of PBMCs with LPS triggered the synthesis of $\mathrm{PGE}_{2}$. We also noticed the formation of other prostanoids such as $\mathrm{PGF}_{2} \alpha$ and thromboxane $\left(\mathrm{TxB}_{2}\right)$, whereas the production of $\mathrm{PGD}_{2}$ remained low (Figure 3A-D). Apparently, a significant inflammatory milieu is required to trigger $\mathrm{PGE}_{2}$ accumulation. We then asked whether selective reduction of $\mathrm{PGE}_{2}$ (mPGES-1 inhibition) as opposed to blocking total prostaglandin production
(COX-2 inhibition) modulates markers of tumor killing in the 3D model. Inhibition of both COX-2 with celecoxib $(\mathrm{Cxb})$ and mPGES-1 with $\mathrm{C} 3$ indeed impaired $\mathrm{PGE}_{2}$ production in PBMC - tumor spheroid cocultures (Figure $3 \mathrm{~A})$. Importantly, whereas $\mathrm{Cxb}$ inhibited the production of all prostanoids tested, $\mathrm{C} 3$ selectively affected $\mathrm{PGE}_{2}$ (Figure 3A-D). Shunting of arachidonic acid towards the production of other prostaglandins did not occur [11].

\section{PGE 2 limits CD80 expression by tumor-associated phagocytes via EP2 and CAMP}

Next, we compared the potency of $\mathrm{Cxb}$ and $\mathrm{C} 3$ to affect phagocyte CD80 expression, which was linked to spheroid killing (Figure 2). Cxb further increased CD80 expression in LPS-challenged tumor-associated phagocytes (Figure 4A). C3 raised LPS-induced CD80 expression to a level comparable to $\mathrm{Cxb}$ (Figure 4A). This suggested $\mathrm{PGE}_{2}$ as a prostanoid responsible for modulating CD80 expression on tumor-associated phagocytes. Incubation of non-activated PBMC spheroid
A

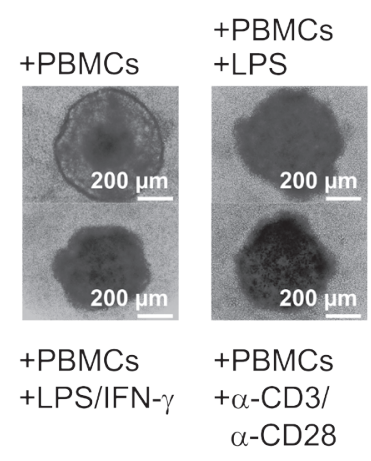

D

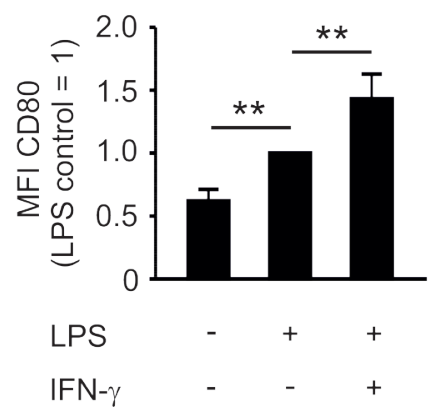

B

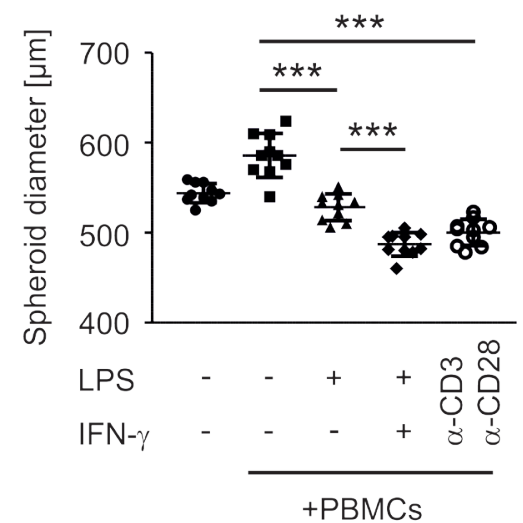

C
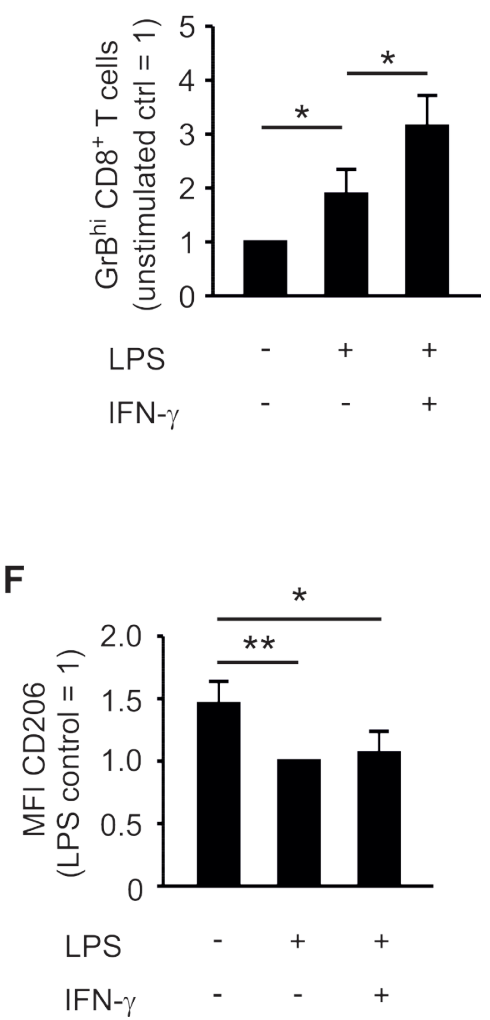

Figure 1: PBMCs require activation to restrict tumor spheroid growth and to upregulate activation markers. PBMCs were pre-stimulated for $30 \mathrm{~min}$ as indicated and then cocultured with MCF-7 tumor spheroids for two days. Spheroid sizes were assessed by microscopy. (A) Photographs of PBMC-spheroid cocultures and (B) diameters of at least nine tumor spheroids are displayed. Data are representatives of three independent experiments. (C) Normalized ratio of $\mathrm{GrB}^{\text {hi }}$ CTLs after two days of coculture is displayed. Data are means \pm SEM of at least four independent donors. Normalized (D) CD80, (E) CD86, and (F) CD206 expression on CD14 CD11 $\mathrm{c}^{+}$ phagocytes is displayed. Data are means \pm SEM of at least four independent donors. $p$-values were calculated using one-way ANOVA (B-F) with Bonferroni's correction. Asterisks indicate significant differences between experimental groups $\left({ }^{*}, p \leq .05,{ }^{* *}, p \leq .01,{ }^{* * *}, p \leq .001\right)$ 
cocultures with $\mathrm{Cxb}$ and $\mathrm{C} 3$ failed to enhance CD80 expression (Figure 4A). CD86 and CD206 expression were not significantly altered by $\mathrm{Cxb}$ or $\mathrm{C} 3$ (Figure 4B,C). Apparently $\mathrm{PGE}_{2}$, but not other prostanoids, selectively blocks activation-induced expression of the co-stimulatory molecule CD80. Importantly, authentic $\mathrm{PGE}_{2}$ dose-dependently counteracted LPS/C3-induced CD80 expression, which was significant at concentrations of $100 \mathrm{ng} / \mathrm{ml}$ (Figure 4D). CD86 expression was not affected (Figure 4E). Surprisingly CD206 expression was also inhibited by $\mathrm{PGE}_{2}$ (Figure 4F). Thus, PGE may impair polarization of tumor-associated phagocytes in general. $\mathrm{PGE}_{2}$ signals through four specific receptors (EP1-4), for which selective agonists are available. Application of the EP2 agonist butaprost, but neither the EP4 agonist cay10580, nor the EP1/3 agonist sulprostone, blocked LPS/C3-induced CD80 expression (Figure 4G). EP2 activation did not affect CD86 expression on phagocytes. However it significantly reduced CD206 expression (Figure 4H,I). EP2 triggers intracellular cAMP accumulation, which is simultaneously degraded by phosphodiesterase-4 (PDE4). Accordingly, increasing intracellular cAMP with the PDE4 inhibitor rolipram significantly counteracted LPS/C3-triggered CD80 expression (Figure 4G), without affecting CD86 (Figure 4H), but reducing CD206 expression (Figure 4I). Taken together, signaling via the EP2/cAMP-pathway

Figure 2: CD80 is needed for the induction of $\mathrm{GrB}^{\text {hi }}$ CTLs and tumor spheroid killing. PBMCs were prestimulated for $30 \mathrm{~min}$ as indicated and subsequently cocultured with MCF-7 tumor spheroids for two days. (A) Normalized ratio of $\mathrm{GrB}^{\text {hi }}$ CTLs after two days of coculture is displayed. Data are means \pm SEM of at least four independent donors. (B) Diameters of at least nine tumor spheroids are displayed. Data are representatives of three independent experiments. $p$-values were calculated using one-way ANOVA with Bonferroni's correction. Asterisks indicate significant differences between experimental groups $(* *, p \leq .01, * * *, p \leq .001)$

A

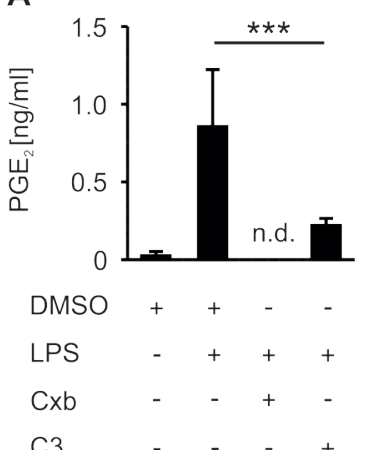

B

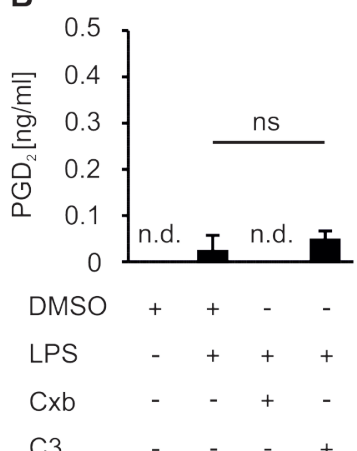

A

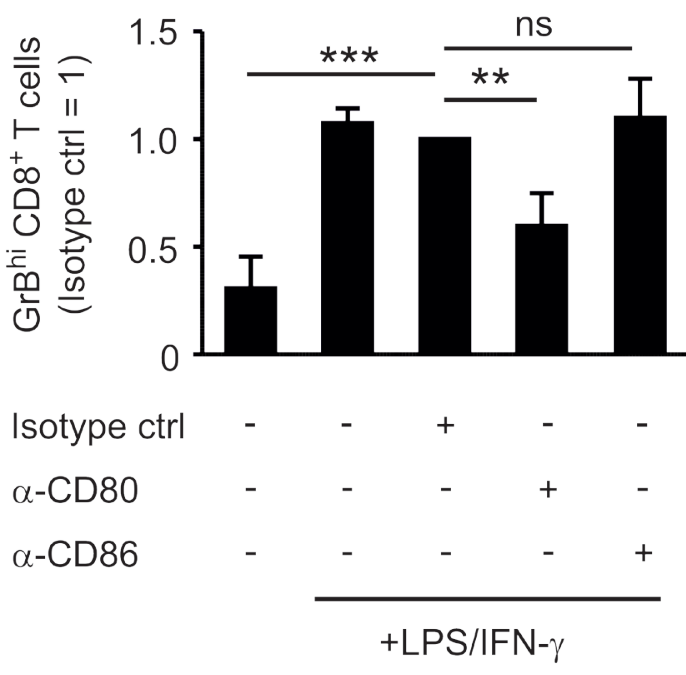

B

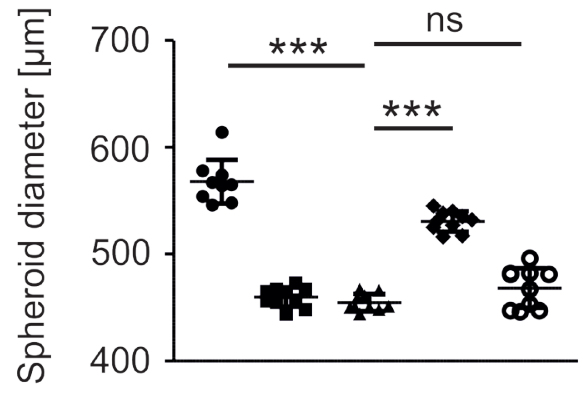

\begin{tabular}{llllll} 
Isotype ctrl & - & - & + & - & - \\
$\alpha-C D 80$ & - & - & - & + & - \\
$\alpha-C D 86$ & - & - & - & - & + \\
\hline
\end{tabular} +LPS/IFN- $\gamma$

C

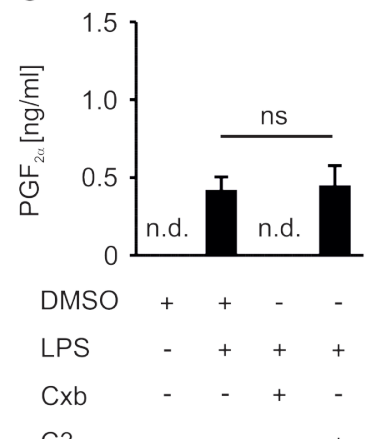

D

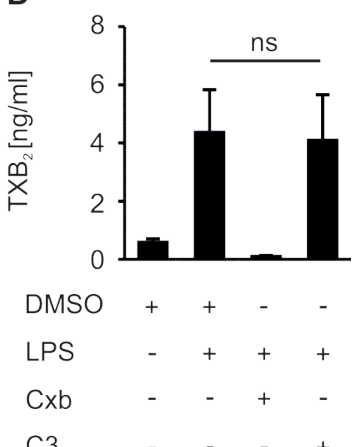

Figure 3: COX-2/mPGES-1-derived PGE, production in PBMC spheroid cocultures. PBMCs were pre-stimulated for 30 min as indicated and subsequently cocultured with MCF-7 tumor spheroids for two days. Cocultures were challenged with the COX-2 inhibitor celecoxib (CxB) or the mPGES-1 inhibitor C3. Prostanoids (A) $\mathrm{PGE}_{2}$, (B) $\mathrm{PGD}_{2},(\mathrm{C}) \mathrm{PGF}_{2} \alpha$, and (D) TxB in supernatants of PBMC MCF-7 spheroid cocultures were measured by LC-MS/MS. Data are means \pm SEM of at least four independent donors. $p$-values were calculated using one-way ANOVA with Bonferroni's correction. Asterisks indicate significant differences between experimental groups $(* * *, p \leq .001)$ 
A
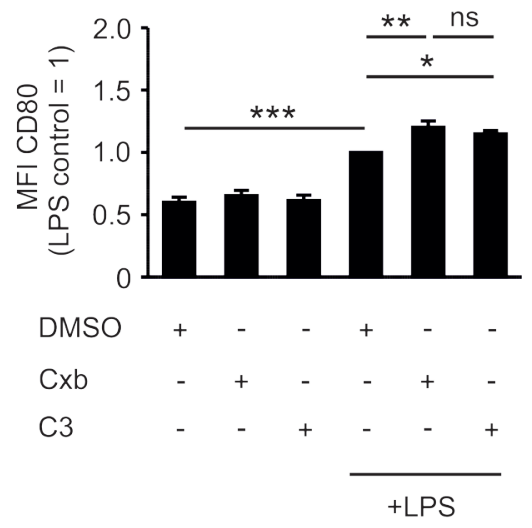

D

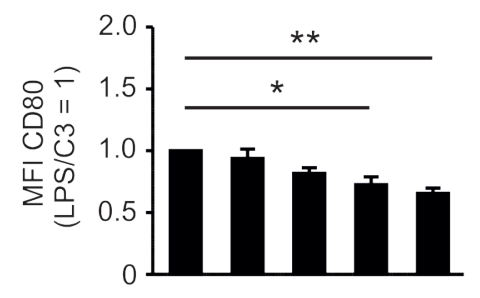

Abs $\mathrm{EtOH}$

$\mathrm{PGE}_{2}[\mathrm{ng} / \mathrm{ml}] \quad \begin{array}{llllll}0 & 10^{0} & 10^{1} & 10^{2} & 10^{3}\end{array}$

$+\mathrm{LPS} / \mathrm{C} 3$

G

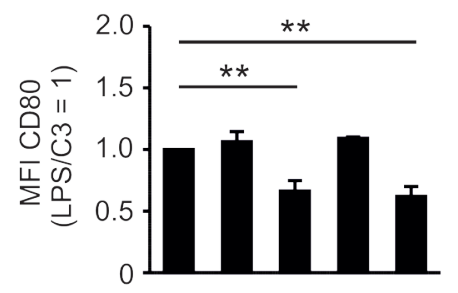

DMSO

EP1/3 agonist

EP2 agonist

EP4 agonist

PDE4 inhibitor

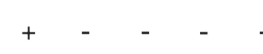

+ LPS/C3
B

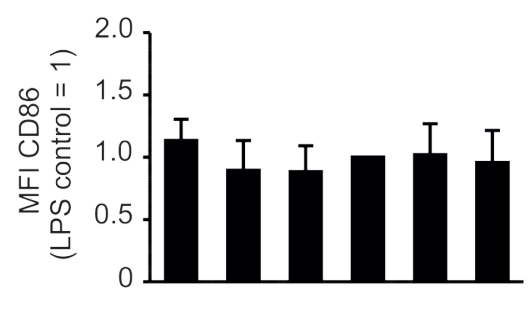

DMSO

Cxb

C3

E

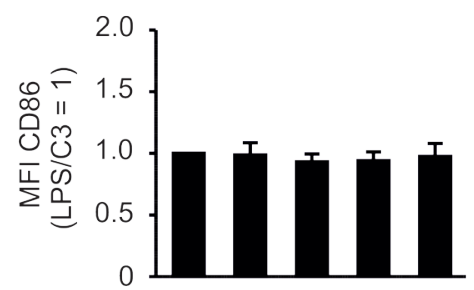

Abs EtOH

$\mathrm{PGE}_{2}[\mathrm{ng} / \mathrm{ml}] \quad \begin{array}{llllll}0 & 10^{0} & 10^{1} & 10^{2} & 10^{3}\end{array}$

$+\mathrm{LPS} / \mathrm{C} 3$

H

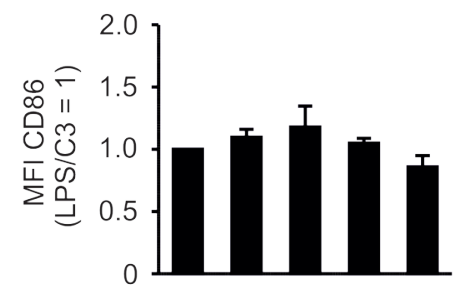

DMSO

EP1/3 agonist

EP2 agonist

EP4 agonist

PDE4 inhibitor
C

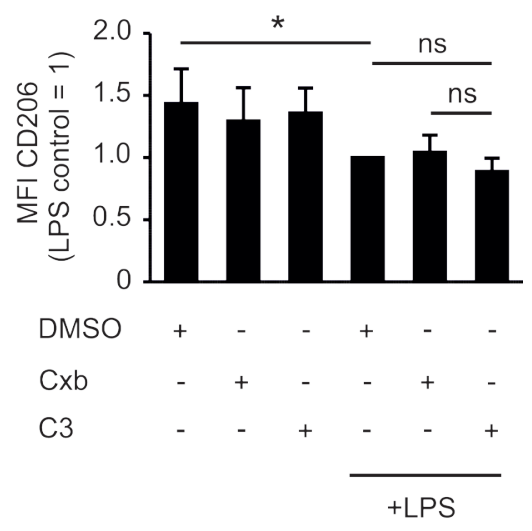

$\mathbf{F}$

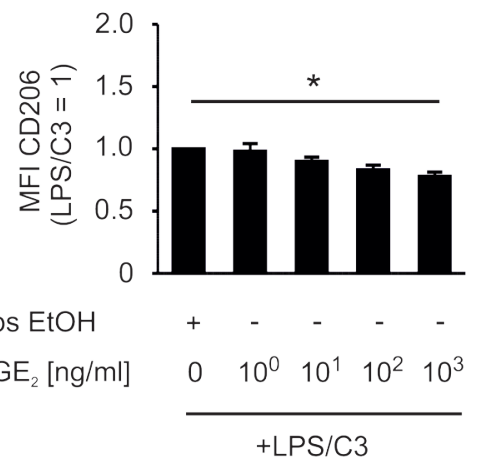

I

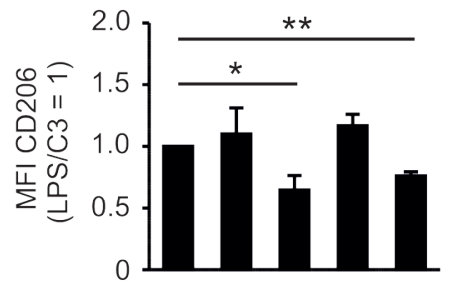

DMSO

EP1/3 agonist

EP2 agonist

EP4 agonist

PDE4 inhibitor

Figure 4: PGE inhibits CD80 and $\mathrm{CD}_{2} 206$ expression of $\mathrm{CD}^{+} \mathbf{C}^{+} \mathrm{CD}_{11 \mathrm{c}^{+}}$phagocytes. PBMCs were pre-stimulated for $30 \mathrm{~min}$ as indicated and subsequently cocultured with MCF-7 tumor spheroids for two days. (A-C) Cocultures were challenged with the COX-2 inhibitor celecoxib (CxB) or the mPGES-1 inhibitor C3. (A) CD80, (B) CD86, and (C) CD206 expression of CD14 ${ }^{+} \mathrm{CD}_{11 \mathrm{c}^{+}}$phagocytes is displayed. Data are means $\pm \mathrm{SD}$ of at least four independent donors. (D-F) Cocultures were dose-dependently challenged with authentic $\mathrm{PGE}_{2}$. (D) CD80, (E) CD86, and (F) CD206 expression of CD14 $\mathrm{CD} 11 \mathrm{c}^{+}$phagocytes is displayed. Data are means $\pm \mathrm{SEM}$ of at least four independent donors. (G-I) Cocultures were challenged with the EP1/3 agonist sulprostone, the EP2 agonist butaprost, the EP4 agonist cay10580, or the PDE4 inhibitor rolipram. (G) CD80, (H) CD86 and (I) CD206 expression of CD14 CD11 ${ }^{+}$phagocytes is displayed. Data are means \pm SEM of at least four independent donors. $p$-values were calculated using one-way ANOVA with Bonferroni's correction. Asterisks indicate significant differences between experimental groups $\left({ }^{*}, p \leq .05,{ }^{* *}, p \leq .01,{ }^{* * *}, p \leq .001\right)$ 
counteracted LPS/C3-induced CD80 expression in tumorassociated human phagocytes and reduced the expression of the anti-inflammatory macrophage marker CD206.

\section{mPGES-1-deficiency delays tumor development in vivo}

We asked whether regulation of parameters affecting tumor killing in the human in vitro setting could be recapitulated in a breast cancer model in vivo. We crossed mPGES-1-deficient mice into the PyMT background, to generate female mice that develop spontaneous breast cancer [20]. Tumors were first observed 8 weeks after birth and tumor development was monitored until sacrifice. MPGES-1-deficiency resulted in strongly reduced $\mathrm{PGE}_{2}$ levels in tumors after sacrifice (20 weeks) (Figure 5A). Importantly, shunting of arachidonic acid towards the production of other prostaglandins was also excluded in PyMT tumors (Figure 5A). Accordingly, both COX1 and COX-2 mRNA were expressed in PyMT tumors, independent of the mPGES-1 status (Figure S3A). Lack of mPGES-1 delayed tumor development and reduced numbers of tumor-bearing mammary glands per mouse compared to WT PyMT mice (Figure 5B). After 20 weeks, loss of mPGES-1 still resulted in significantly reduced tumor mass (Figure 5C). However, the relative distribution of tumor size in mPGES-1-deficient PyMT mice was not different from WT PyMT mice (Figure 5D). Apparently, the absence of $\mathrm{PGE}_{2}$ enhances tumor dormancy rather than affecting tumor growth kinetics. This observation might indicate a role of mPGES-1-derived $\mathrm{PGE}_{2}$ in modulating tumor immune escape [27].

\section{Tumor-associated macrophage content and activation is altered in $\mathbf{m P G E S}-\mathbf{1}^{-/-}$tumors}

We asked whether reduced tumor outgrowth was correlated with a different immune status in the tumors. Hence, tumor single cell suspensions were analyzed by polychromatic FACS to characterize tumor-infiltrating

\section{C}

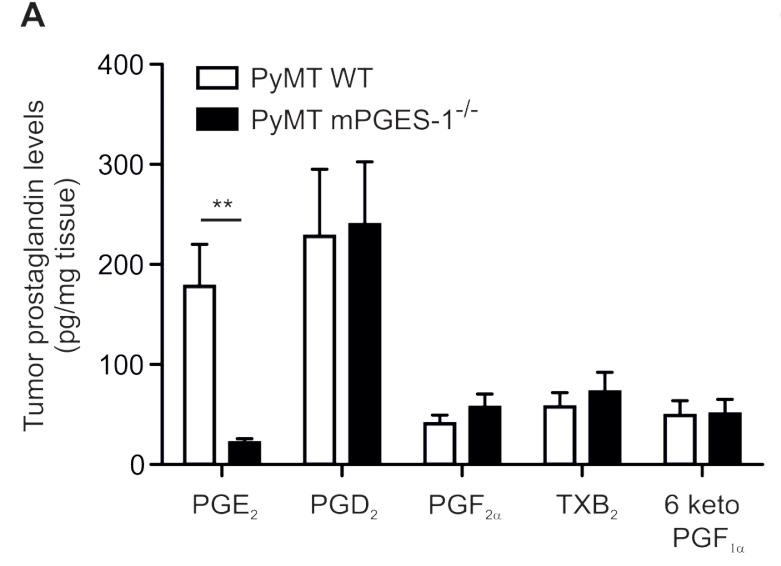

B

D
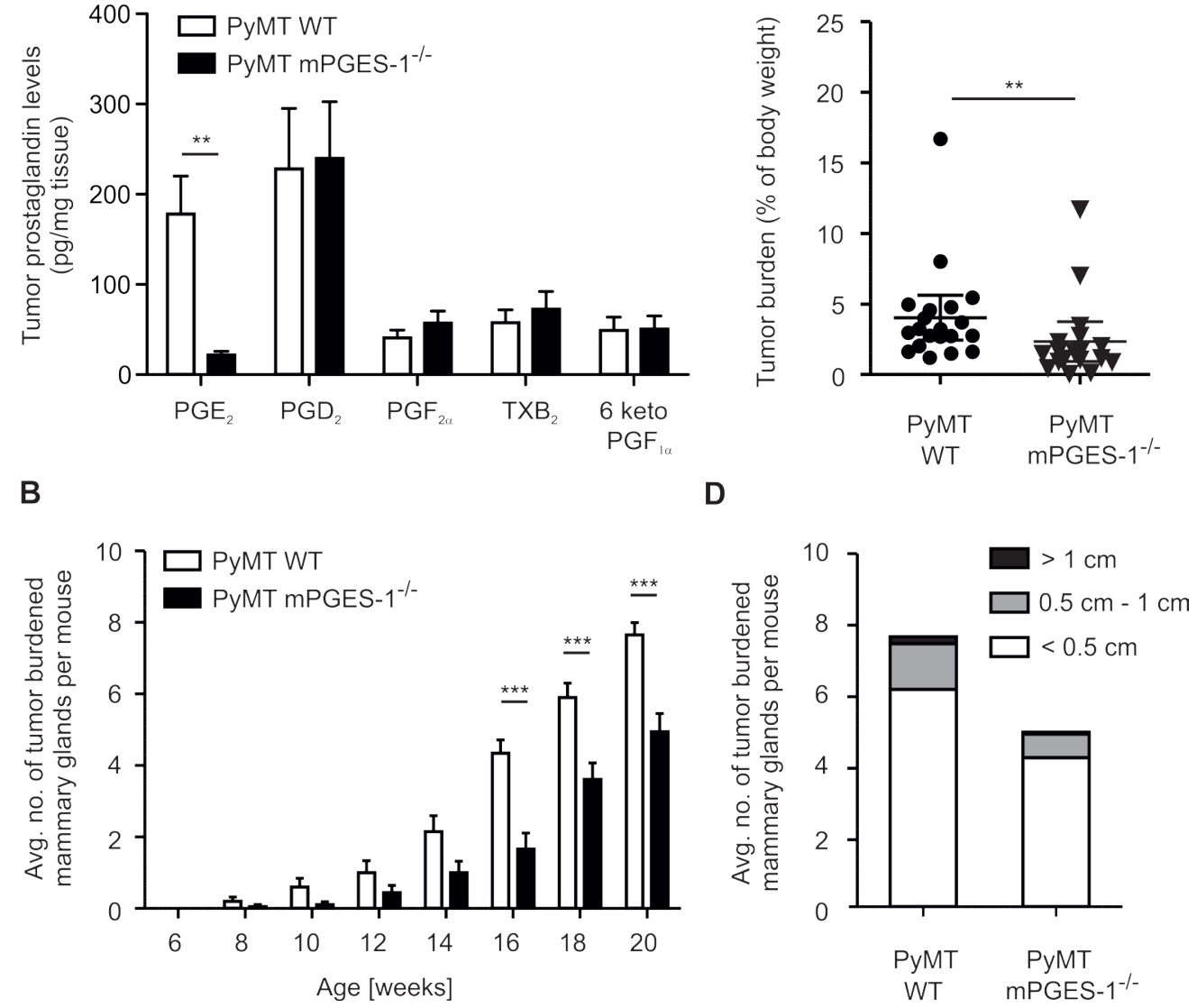

Figure 5: mPGES-1-deficiency impairs PyMT tumor growth. (A) Prostanoids $\mathrm{PGE}_{2}, \mathrm{PGD}_{2}, \mathrm{PGF}_{2} \alpha$, TxB 2 , and 6 keto PGF $\alpha$

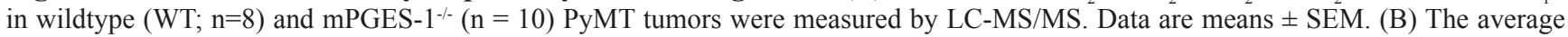
number of tumor-bearing mammary glands per mouse is displayed. (C,D) PyMT mice were sacrificed 20 weeks after birth. (C) Tumor burden is defined as the ratio of total tumor mass and mouse body weight. (D) Tumors were categorized and their respective numbers are displayed. (B-D) Data are means \pm SEM of 20 WT and 18 mPGES- $1^{-/}$mice. $p$-values were calculated using student's t-test (C) or two-way ANOVA with Bonferroni's correction (A,B). Asterisks indicate significant differences between experimental groups $(*, p \leq .05, * *, p \leq .01$, $* * *, p \leq .001)$ 
leukocyte populations [25]. There was a tendency of increased leukocyte infiltration into mPGES-1-deficient tumors (Figure 6A). Phagocyte subpopulations in PyMT tumors are mainly $\mathrm{F} 4 / 80^{+} \mathrm{CD} 11 \mathrm{~b}^{\text {low }} \mathrm{CD} 11 \mathrm{c}^{+}$tumorassociated macrophages (TAMs), CD11 $\mathrm{b}^{\text {high }} \mathrm{F} 4 / 80^{+}$ resident macrophages, and $\mathrm{F} 4 / 80^{\text {low }} \mathrm{CD} 11 \mathrm{c}^{+} \mathrm{MHCII}^{+} \mathrm{DCs}$ (Figure S1C) [28]. Furthermore, monocytes, neutrophils, and different lymphocyte populations are present (Figure $6 \mathrm{~B})$. In general, TAMs promote tumor growth and their abundance at the tumor site is often indicative of bad prognosis in breast cancer patients [29]. To our surprise, the relative content of TAMs was significantly increased in PyMT tumors of mPGES-1 $1^{-/}$mice (Figure 6B). Thus, the usual correlation between breast tumor growth and the number of tumor infiltrating phagocytes was not reflected in our system. We hypothesized that, in agreement with our in vitro observations, polarization of TAMs in mPGES-1 ${ }^{-/}$tumors might explain the differences in tumor growth. Therefore, polarization of phagocytes in WT and mPGES-1-deficient tumors was assessed using flow cytometry. When analyzing the immunophenotype of TAMs, we indeed observed an increase in CD80 expression in mPGES-1 ${ }^{-/}$TAMs (Figure 6C). CD86 and
CD206 expression in TAMs remained unchanged (Figure $6 \mathrm{D}, \mathrm{E})$. However, we observed reduced CD206 expression by resident macrophages in $\mathrm{mPGES}-1^{-/}$tumors. These data indicate a different impact of $\mathrm{PGE}_{2}$ on resident and monocyte-derived macrophages [28].

\section{MPGES-1-deficiency enhances phagocyte- dependent CTL activation}

Following the hypothesis that a reduced occurrence of mPGES-1 ${ }^{-/}$PyMT tumors was due to enhanced immune control, we wondered if elevated CD80 expression in mPGES-1/- murine macrophages translates into CTL activation. First, we asked whether enhanced CD80 was observed in mPGES-1 ${ }^{-/}$BMDM stimulated with LPS/ IFN- $\gamma$ compared to WT macrophages. Interestingly, mPGES-1 ${ }^{-/}$BMDM, which barely produced $\mathrm{PGE}_{2}$, did not show alterations in CD80 or CD86 induction compared to WT BMDM after stimulation with LPS/ IFN- $\gamma$ (Figure S3C,D). Thus, not only an activating stimulus per se, but also the tumor milieu might be necessary to reveal immune modulating effects of $\mathrm{PGE}_{2}$.
A
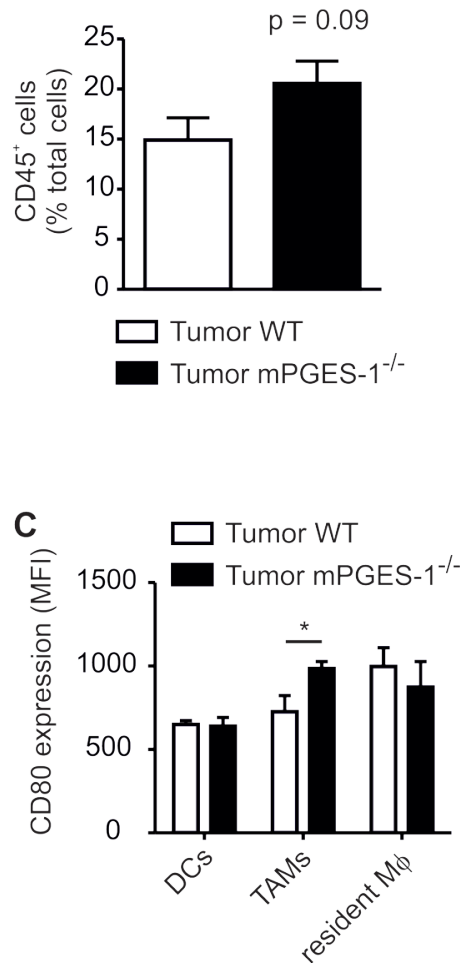

B
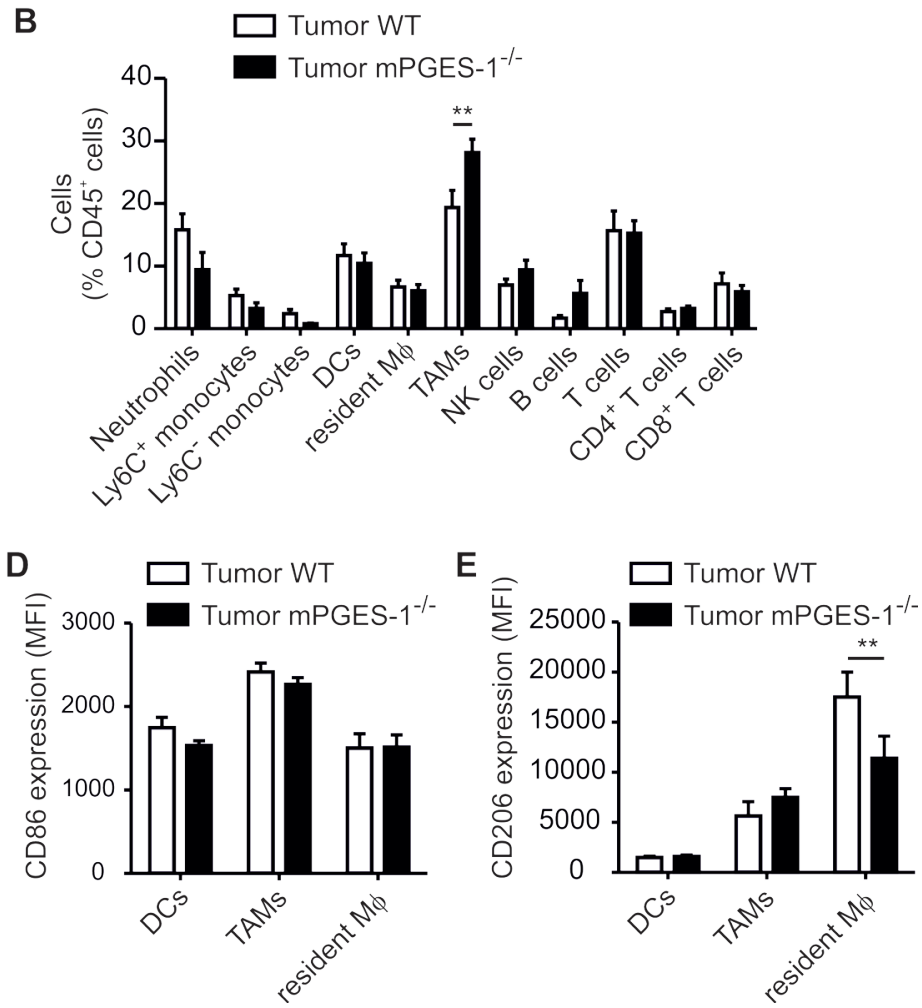

Figure 6: mPGES-1-deficiency increases the abundance of F4/80 ${ }^{+}$CD11 $^{+}$phagocytes in PyMT tumors. PyMT mice were sacrificed 20 weeks after birth. Immune cell subsets and activation markers in PyMT tumors were determined by flow cytometry. (A) The relative content of $\mathrm{CD}_{4} 5^{+}$immune cells in tumors of wildtype (WT) and mPGES-1/- tumors is shown. (B) The relative amount of immune cell subsets is displayed. Data are means \pm SEM of 11 WT and 7 mPGES- $1^{-/}$mice. (C) CD80, (D) CD86, and (E) CD206 expression by tumor-associated macrophages (TAMs), dendritic cells (DCs), and resident macrophages (resident M $\varphi$ ) in PyMT tumors is displayed. Data are means \pm SEM of 6 WT and 7 mPGES- $1^{-/}$mice. Exact $p$-values were calculated using student's t-test (A), otherwise $p$-values were calculated using two-way ANOVA with Bonferroni's correction (B-E). Asterisks indicate significant differences between experimental groups $(*, p \leq .05, * *, p \leq .01)$. 
These selective immune modulating effects of $\mathrm{PGE}_{2}$ might be most apparent in a set-up that comprises both inflammatory (LPS/IFN- $\gamma$ ) as well as anti-inflammatory (breast tumor microenvironment) components. To test this hypothesis we generated tumor spheroids of E0771 murine mammary carcinoma cells. These spheroids were incubated with LPS/IFN- $\gamma$-stimulated WT or mPGES-1 ${ }^{-/}$ bone marrow monocytes for $24 \mathrm{~h}$, followed by addition of OVA SIINFEKL as a model antigen and spleen-derived OT-I CTLs for another 4 days. These T cells specifically recognize the OVA SIINFEKL peptide, resulting in a model of antigen-specific CTL activation in the tumor microenvironment. In this setting, mPGES-1/- monocytederived tumor spheroid-infiltrating macrophages indeed expressed higher CD80, but not CD86, levels compared to wildtype macrophages (Figure 7A,B). These data corroborated our findings in the PyMT model. Spheroid-infiltrating OT-I cells displayed higher rates of proliferation (Figure 7C,D) and GrB expression (Figure 7E) when spheroids contained mPGES- $1^{-/}$monocytederived macrophages compared to WT macrophages. These data support the notion that reduced tumor growth in $\mathrm{mPGES}-1^{-/}$PyMT mice was due to enhanced immune control. Importantly, infiltration of pre-activated WT or mPGES- $1^{-/}$spleen CTLs did not affect $\mathrm{T}$ cell proliferation

A

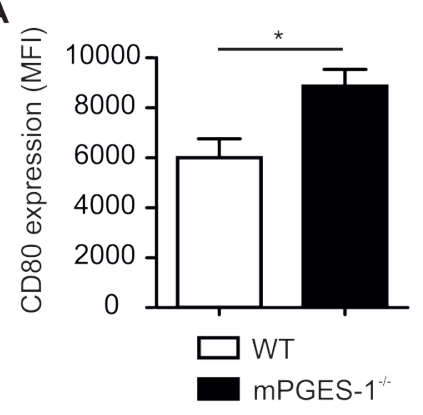

E

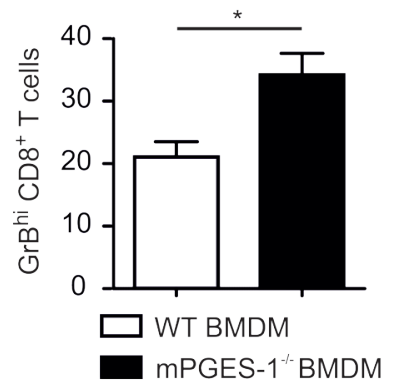

B

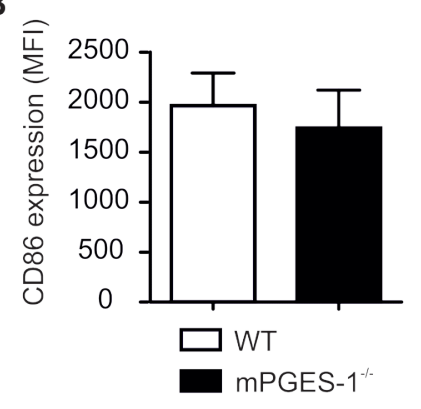

$\mathbf{F}$

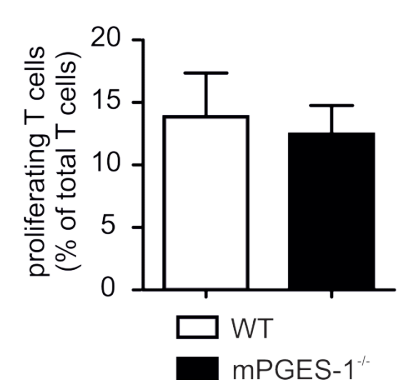

or GrB expression (Figure 7F,G). These novel data demonstrate that mPGES-1-deficiency does not affect basic CTL function. Thus, a direct impact of mPGES-1deficiency on CTL function in PyMT tumors was unlikely contributing to the phenotype of mPGES- $1^{-/}$PyMT mice. We conclude that mPGES-1/- TAMs support CTL activation. This might be an important mechanism in restricting tumor occurrence in mPGES-1/- PyMT mice.

\section{DISCUSSION}

In our in vitro coculture tumor model, non-activated PBMCs were unable to kill tumor spheroids. Rather, they required TLR4 activation to increase CD80 expression and to concomitantly elevate $\mathrm{GrB}^{\text {hi }}$ CTL numbers. CD80 appeared not only as a signature marker, but also a functional prerequisite for immune activation in this model. Along these lines, blocking CD80 reduced the number of $\mathrm{GrB}^{\text {hi }} \mathrm{CTL}$ and prevented tumor spheroid killing. CD86 expression was not regulated by LPS and blocking of CD86 neither changed $\mathrm{GrB}^{\text {hi }}$ CTL numbers nor rescued tumor spheroid sizes. However, in the human system other phagocyte subsets might regulate CD86 expression in response to LPS. For instance, monocyte differentiation with human plasma generates $\mathrm{CD} 14^{+}$

\section{C}

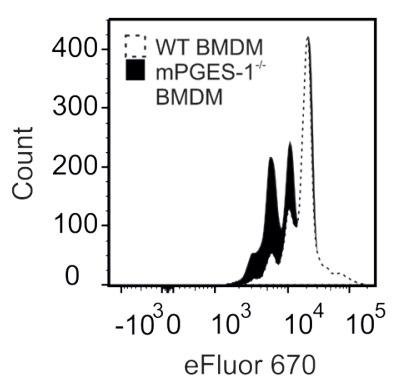

G

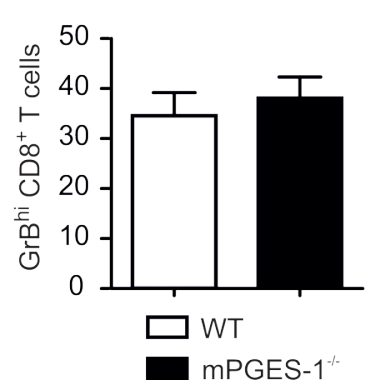

D

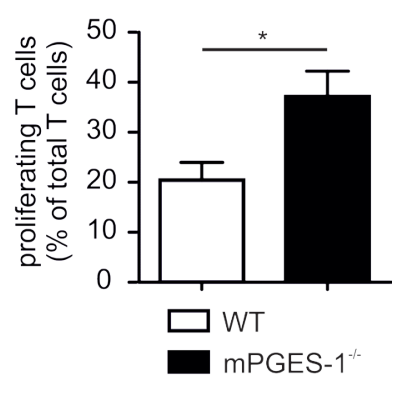

Figure 7: MPGES-1-deficiency promotes CTL activation by tumor-associated macrophages. (A-E) Bone marrow monocytes of WT or mPGES-1 1 mice were pre-stimulated with LPS/IFN- $\gamma$ for $30 \mathrm{~min}$ and cocultured with E0771 tumor spheroids for $24 \mathrm{~h}$. Afterwards, OVA SIINFEKL peptide was added for $1 \mathrm{~h}$ followed by addition of spleen-derived OT-I CTLs for another 4 days. Afterwards cocultures were analyzed by flow cytometry. (A) CD80 and (B) CD86 expression of CD11 ${ }^{+} \mathrm{F} 4 / 80^{+}$monocyte-derived macrophages (BMDM) is displayed. Data are means \pm SEM of four independent experiments. (C) A representative histogram and (D) a quantification of proliferating OT-I T cells is displayed. (E) The relative amount of $\mathrm{GrB}^{\mathrm{hi}} \mathrm{CTLs}$ is shown. Data are means $\pm \mathrm{SEM}$ of four independent experiments using cells of 4 WT and 4 mPGES-1 1 mice. (F,G) Pre-activated spleen-derived CTLs of WT or mPGES-1 1 mice were cocultured with E0771 tumor spheroids for 3 days. (F) The relative amount of proliferating $\mathrm{T}$ cells and $(\mathrm{G})$ the relative amount of $\mathrm{GrB}^{\text {hi }} \mathrm{CTLs}$ is shown. Data are means \pm SEM of four independent experiments using cells of $4 \mathrm{WT}$ and $4 \mathrm{mPGES}-1 \stackrel{-1}{ }$ mice. $p$-values were calculated using student's t-test. Asterisks indicate significant differences between experimental groups $\left({ }^{*}, p \leq .05\right)$. 


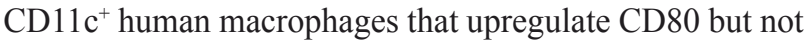
CD86, and a minor population of CD14- CD11 ${ }^{+}$cells that selectively upregulate CD86 upon LPS stimulation (data not shown).

Our in vitro model allowed investigating the immune modulating function of $\mathrm{PGE}_{2}$ in an authentic human tumor context. These functions were re-capitulated in the murine system both in vitro and in vivo. Due to the high reproducibility, this model might be easily adaptable for high-throughput analysis of compounds affecting tumor immunity. In this study, inhibition of mPGES-1 by C3 selectively inhibited $\mathrm{PGE}_{2}$ production. Importantly, it did not provoke prostanoid shunting as previously observed in LPS/C3-stimulated mouse peritoneal macrophages [11]. Shunting was also not observed in mPGES-1 ${ }^{-/}$PyMT tumors. Either selective inhibition of $\mathrm{PGE}_{2}$ with $\mathrm{C} 3$ or inhibition of total prostanoid production with $\mathrm{Cxb}$ enhanced CD80 expressed on spheroidinfiltrating phagocytes. Thus, $\mathrm{PGE}_{2}$ was the relevant prostanoid responsible for modulating CD80 expression. Moreover, at least in the in vitro system, mPGES-1 inhibition recapitulated the effect of COX2 inhibition. Addition of exogenous $\mathrm{PGE}_{2}$, the EP2 receptor agonist butaprost, or the PDE4 inhibitor rolipram revealed that the $\mathrm{PGE}_{2} / \mathrm{EP} 2 / \mathrm{cAMP}$ pathway impaired both CD80 and CD206 expression on phagocytes. We conclude that $\mathrm{PGE}_{2}$ signaling alters polarization of human tumor-associated phagocytes independent of the M1/M2 paradigm [30]. In line, $\mathrm{PGE}_{2}$ inhibited inflammatory macrophage function in numerous inflammation models through EP2/EP4 and elevated intracellular cAMP [31-33].

Modulation of costimulatory molecule expression by $\mathrm{PGE}_{2}$ requires the tumor scenario as $\mathrm{CD} 80$ expression of $\mathrm{mPGES}^{-1 /-}$ BMDMs was similar to WT BMDMs in monocultures. Only when infiltrating tumor spheroids mPGES-1 ${ }^{-/}$macrophages expressed elevated CD80 levels. Similarly, mPGES-1/- GM-CSF/IL-4-differentiated bone marrow-derived dendritic cells (BMDCs) did not show altered levels of CD80 and CD86 after LPS/IFN- $\gamma$ challenge, whereas EP2/cAMP signaling rather enhanced CD80 and CD86 expression in TNF- $\alpha$-differentiated BMDCs [16, 34]. In BMDMs, LPS-triggered CD40 expression was inhibited by the addition of $\mathrm{PGE}_{2}, \mathrm{CD} 80$ expression remained unaffected, while CD86 was induced [35]. However, $\mathrm{PGE}_{2}$ inhibited CpG-induced CD80 expression and IFN $-\alpha$ secretion in human plasmacytoid dendritic cells via EP2 and EP4 signaling [36]. These findings suggest that regulation of costimulatory molecules in monocultures of BM-derived cells depends on the cell type, stimulus, and differentiation protocols. Principally, mPGES-1-deficiency does not seem to alter functional outcomes in phagocyte monocultures. We propose that monoculture models are suitable to investigate the induction of signaling molecules such as CD80/CD86. However, their fine-tuning often relies on more complex interactions e.g. a crosstalk of different cell types and/or the presence of tumor environmental factors. These factors are only available in tumor spheroid immune cell cocultures or in vivo tumor models. For instance, regulatory $\mathrm{T}$ cells may be involved in the regulation of CD80 and CD206 expression of phagocytes. PGE promotes Treg activity, which can directly regulate CD80/ CD86 expression [37, 38].

In MMTV/PyMT mice, we observed that the loss of mPGES-1 delayed breast cancer development. In a different breast cancer model, Her2/c-neu mice, genetic depletion of mPGES-1 reduced mammary tumorigenesis, aromatase activity, and angiogenesis [39]. However, the impact on tumor-associated immunity was not investigated. We hypothesize that delayed breast tumor development in our model was due to prolonging tumor dormancy, indicating a potential of mPGES-1 inhibitors for tumor prophylaxis. An explanation for enhanced tumor dormancy might be a superior tumor immune control by CTLs, similar to the situation observed in our in vitro murine model (that mimics early tumors). Nevertheless, we observed higher expression of CD80, a feature of immune control, also in advanced tumors. This feature may be more pronounced or relevant in small, dormant malignancies. Accordingly, although CD80-expressing mPGES- $1^{-/}$macrophages more efficiently activated CTLs in vitro, the $\mathrm{T}$ cell infiltrate in established mPGES-1/PyMT tumors was not altered. Thus, our data indicate that a deficient mPGES-1-dependent $\mathrm{PGE}_{2}$ production alone was insufficient to lift immune suppression from established PyMT tumors. In vitro, high expression of CD80 on human and murine phagocytes required TLR stimulation. Indeed, the FDA recently approved the TLR2/TLR4 agonist Bacillus Calmette-Guérin (BCG), the TLR2/TLR4 agonist monophosphoryl lipid A (MPL) and the TLR9 agonist imiquimod as anti-tumor agents [3]. The tumor vaccine L-BLP25, which contains an MPL component, failed to increase overall survival in a Phase III clinical trial in non-small cell lung carcinoma (NSCLC) patients. However, it showed a significant improvement in patients treated with low-dose cyclophosphamide, which is known to abrogate tolerance of regulatory $\mathrm{T}$ cells in tumor patients $[40,41]$. Together with our findings we hypothesize that TLR agonists may not lack efficiency in general but their original anti-tumoral mode of action may be restricted by negative feedback mechanisms dependent, among others, on $\mathrm{PGE}_{2}$. Thus, the development of mPGES-1 inhibitors as natural combinational therapy partners for TLR agonists may be relevant to enhance the efficiency of TLR agonists as cancer therapeutics. 


\section{MATERIALS AND METHODS}

\section{Materials}

The mPGES-1 inhibitor compound III (C3) (5 $\mu \mathrm{M})$ [11] was synthesized by NovaSAID AB (Solna, Sweden). Celecoxib $(5 \mu \mathrm{M})$, lipopolysaccharide (LPS) $(50 \mathrm{ng} / \mathrm{ml})$, dimethylsulfoxide (DMSO), and rolipram $(10 \mu \mathrm{M})$ were purchased from Sigma-Aldrich (St. Louis, MO, USA). CD80 $(5 \mu \mathrm{g} / \mathrm{ml})$ and CD86 $(5 \mu \mathrm{g} /$ $\mathrm{ml})$ neutralizing antibodies and the isotype control (5 $\mu \mathrm{g} / \mathrm{ml}$ ) were purchased from eBiosciences (Frankfurt, Germany) or R\&D Systems (Minneapolis, MN, USA). PGE $_{2}$, butaprost $(5 \mu \mathrm{M})$, sulprostone $(5 \mu \mathrm{M})$ and cay 10580 $(200 \mathrm{nM})$ were from Cayman Chemicals (Ann Arbor, MI, USA). Both human and murine interferon- $\gamma$ (IFN- $\gamma$ ) (100 U/ml) were from Peprotech (Hamburg, Germany). Anti-CD3/anti-CD28 Dynabeads (1:20 - bead: PBMCs) were from Invitrogen (Carlsbad, Germany). OVA (257267) SIINFEKEL was from Anaspec (Seraing, Belgium). Cell proliferation dye eFluor670 was from eBiosciences. Matrigel was from Corning Life Sciences (Amsterdam, Netherlands).

\section{Mouse strains and genotyping}

All procedures involving mice followed the guidelines of the Hessian animal care and use committee. Wildtype (WT) and mPGES- $1 /$ mice with or without crossing into a PyMT background as well as Rag1 knockout/transgenic OT-I T cell receptor (OT-I) mice (all C57BL/6) were used. For genotyping, tail-tips were lysed with KAPA Genotyping lysis buffer (Peqlab, Erlangen, Germany) and resulting DNA solutions were analyzed with PCR amplification using KAPA Hotstart Genotyping reaction mix (Peqlab) and standard protocols. Primers a and $b$ were used for the wildtype allele and primers $b$ and $\mathrm{c}$ were used for the mutated allele. 'a': 5'-CAG TAT TAC AGG AGT GAC CCA GAT GTG-3' (specific for targeted mPGES-1 gene). 'b': 5'-GGA AAA CCT CCC GGA CTT GGT TTT CAG-3' (specific for the mPGES-1 gene downstream of the targeting construct). 'c': 5'-ATC GCC TTC TAT CGC CTT CTT GAC GAG-3' (specific for the neo resistance gene). Primers used for PyMT genotyping include PyMT forward: 5'-CTA GGC CAC AGA ATT GAA AGA TCT-3' and PyMT reverse: 5'-GTA GGT GGA AAT TCT AGC ATC ATC C-3'. Internal control forward: 5'-GGA AGC AAG TAC TTC ACA AGG G-3' and internal control reverse: 5'-GGA AAG TCA CTA GGA GCA GGG-3'.

\section{Cell culture and MCTS generation}

MCF-7 breast carcinoma cells were purchased from ATCC-LGC Standard GmbH (Wesel, Germany). E0771 cells were from CH3 Biosystems (Amherst, NY, USA). Cells were cultured in RPMI 1640, supplemented with $5 \mathrm{mM}$ glutamine, $100 \mathrm{U} / \mathrm{ml}$ penicillin, $100 \mu \mathrm{g} / \mathrm{ml}$ streptomycin as well as 10\% heat-inactivated FCS and were maintained at $37^{\circ} \mathrm{C}$ in a humidified atmosphere with $5 \% \mathrm{CO}_{2}$. Spheroids were generated from MCF-7 cells using the liquid overlay technique as described [21]. Spheroids generated from E0771 cells were initiated using $5 \times 10^{4}$ cells $/ \mathrm{ml}$ and were cultured in cell-repellent 96-well plates (Greiner Bio-One GmbH, Frickenhausen, Germany) with a final concentration of $1,5 \%$ matrigel [22].

\section{Isolation of human PBMCs}

PBMCs were isolated from Buffy Coats of anonymous healthy donors obtained from DRK Blutspendedienst (Frankfurt, Germany) using Ficoll gradient centrifugation [23].

\section{Isolation of murine $\mathrm{CD8}^{+} \mathrm{T}$ cells and monocytes}

Spleens were isolated from WT, mPGES- $1^{-/}$, and OT-I mice. Single cell suspensions were generated by processing the spleen through a $70 \mu \mathrm{m}$ nylon mesh (BD Biosciences, Heidelberg, Germany) with a syringe plug. After performing $\mathrm{RBC}$ lysis, $\mathrm{CD}^{+} \mathrm{T}$ cells were isolated using the mouse $\mathrm{CD}^{+} \mathrm{T}$ cell Isolation Kit (Stemcell Technologies, Cologne, Germany) and stained with the Cell Proliferation Dye eFluor670. Bone marrow was isolated from tibia and femur of WT and mPGES-1mice. After RBC lysis, monocytes were isolated using the Monocyte Isolation Kit and the AutoMACS separator (Miltenyi Biotec GmbH, Bergisch Gladbach, Germany).

\section{PBMC MCF-7 tumor spheroid cocultures}

Media of MCF-7 spheroids were changed prior to PBMC addition. PBMCs were either left untreated or stimulated with $50 \mathrm{ng} / \mathrm{ml}$ LPS or LPS and $100 \mathrm{U} /$ $\mathrm{ml}$ IFN- $\gamma$. Neutralizing antibodies or inhibitors were immediately added after activation of PBMCs. PBMCs were then cocultured with MCF-7 tumor spheroids for the times indicated. Tumor spheroid size was determined as described recently [21].

\section{E0771 tumor spheroid cocultures}

For E0771 spheroid CD8 ${ }^{+} \mathrm{T}$ cell cocultures media were changed prior to the addition of $1 \times 10^{5} \mathrm{CD} 8^{+} \mathrm{T}$ cells 
per spheroid. $\mathrm{CD} 8^{+} \mathrm{T}$ cells were either left untreated or stimulated with anti-CD3/anti-CD28 Dynabeads (1:1 bead:CD8 ${ }^{+} \mathrm{T}$ cell) for $30 \mathrm{~min}$. E0771 spheroid mPGES-1/and wildtype $\mathrm{CD} 8^{+} \mathrm{T}$ cell cocultures were maintained for 3 days. For cocultures of E0771 spheroids with monocytes and $\mathrm{CD}^{+}$OT-I T cells, $1 \times 10^{5}$ monocytes prestimulated with $50 \mathrm{ng} / \mathrm{ml} \mathrm{LPS}$ and $100 \mathrm{U} / \mathrm{ml}$ IFN- $\gamma$ were added to each spheroid. $24 \mathrm{~h}$ after monocyte addition, cocultures were stimulated with OVA SIINFEKEL for $1 \mathrm{~h}$, followed by addition of $1 \times 10^{5}$ OT-I CD8 ${ }^{+} \mathrm{T}$ cells per spheroid. Cocultures were further maintained for 4 days.

\section{Prostanoid quantification by liquid chromatography-tandem mass spectrometry (LC- MS/MS)}

LC-MS/MS analysis of $\mathrm{PGF}_{2} \alpha, \mathrm{PGE}_{2}, \mathrm{PGD}_{2}$, and $\mathrm{TXB}_{2}$ from coculture supernatants and PyMT tumors was performed after solid-phase extraction and analysis was performed as previously described [24].

\section{Flow cytometry}

Samples were acquired with a LSRII/Fortessa flow cytometer (BD Biosciences, Heidelberg, Germany) and analyzed using FlowJo software 7.6.1 (Treestar, Ashland, OR, USA) or FACSDiva (BD). All antibodies and secondary reagents were titrated to determine optimal concentrations. CompBeads (BD) were used for singlecolor compensation to create multi-color compensation matrices. For gating, fluorescence minus one (FMO) controls were used. The instrument calibration was controlled daily using Cytometer Setup and Tracking beads (BD). For staining of human spheroid-infiltrating phagocytes, cell suspensions were blocked with Fc Receptor Binding Inhibitor (eBioscience) for $15 \mathrm{~min}$ on ice and stained with anti-CD45-PE, anti-CD11c-V450, anti-CD14-APC-H7, anti-CD86-PE, anti-CD206-PE-Cy5 (BD), and anti-CD80-APC (eBioscience) for $15 \mathrm{~min}$ on ice. For murine spheroid-infiltrating phagocytes, antiCD11b-eFluor605NC (ebioscience), anti-F4/80-PE-Cy7 (Biolegend), anti-CD80-APC and anti-CD86-PE (both $\mathrm{BD})$ were used. For intracellular staining of GrB in human CTLs, non-infiltrating PBMCs were fixed with Cytofix/ Cytoperm buffer (BD) for a maximum of $5 \mathrm{~min}$ on ice, followed by washing and permeabilization with Perm/ Wash buffer (BD) as described [21]. Permeabilized cells were blocked with Fc Receptor Binding Inhibitor for 15 min on ice and GrB expression in CTLs was determined using anti-CD45-APC, anti-CD4-FITC, anti-GrB-PE (Immunotools, Friesoythe, Germany), anti-CD3-V450, anti-CD8-APC-Cy7 (BD). For mouse spheroid-associated CTLs, anti-CD3-PE-CF594 and anti-CD8-BV650 (both from BD) as well as anti-GrB-PE (ebioscience) were used. Characterization of PyMT tumor infiltrating leukocytes was performed essentially as described previously [25]. The following antibodies were used: anti-CD3-PE-CF594, anti-CD4-V500, anti-CD11c-AlexaFluor700, anti-CD19APC-H7, anti-Ly6C-PerCP-Cy5.5 (all from BD), antiCD8-eFluor650, anti-CD11b-eFluor605NC (both from eBioscience), anti-CD45-VioBlue, anti-CD49b-PE, antiMHC-II-APC (all three from Miltenyi), anti-F4/80-PECy7, anti-Ly6G-APC-Cy7, anti-SiglecH-FITC (all three from Biolegend, San Diego, CA, USA). To characterize phagocyte subset activation, PyMT tumor single cell suspensions were stained with anti-CD45-VioBlue (Miltenyi), anti-F4/80-PE-Cy7, anti-CD11c-PerCP-Cy5.5, anti-Ly6G-APC-Cy7 (Biolegend), anti-CD80-APC, antiCD86-PE (BD), and anti-CD206-FITC (Biolegend).

\section{Screening of PyMT tumors}

Female PyMT ${ }^{+/-}$were screened from week 6 after birth for breast tumors. Tumor size (by caliper) and localization were monitored.

\section{Tissue isolation from PyMT mice and generation of single cell tumor suspensions}

20 weeks after birth, PyMT mice were sacrificed and perfused with PBS. After perfusion, PyMT tumors and spleen were isolated and their respective weight was recorded. Tissues were lysed with the Miltenyi Tumor dissociation kit and the GentleMACS (Miltenyi, Bergisch Gladbach, Germany) using standard protocols.

\section{Statistical analysis}

All data represented in graphs are means \pm SEM unless stated otherwise. Statistically significant differences between groups were calculated using student's t-test or ANOVA with Bonferroni's post-correction and were considered significant if $*, p \leq 0.05,{ }^{* *}, p \leq 0.01,{ }^{* * *}, p$ $\leq 0.001$.

\section{ACKNOWLEDGMENTS}

We thank Prof. Dr. S. Akira (Department of Host Defense, Osaka University, Japan) for providing the mPGES-1-deficient mice and Magarete Mijatovic, Gudrun Beyer, and Franz Streb for excellent technical assistance. This work was supported by Deutsche Forschungsgemeinschaft (SFB 1039, Excellence Cluster Cardiopulmonary Systems), Deutsche Krebshilfe (111578, 110637), Sander Foundation (2013.036.1), and Else Kröner-Fresenius-Siftung (Else Kröner-FreseniusGraduiertenkolleg and Translational Research Innovation Pharma, TRIP). The authors declare that they have no conflicts of interest. 


\section{CONFLICTS OF INTEREST}

The authors declare that they have no conflicts of interest.

\section{REFERENCES}

1. Mellman I, Coukos G and Dranoff G. Cancer immunotherapy comes of age. Nature. 2011; 480(7378):480-489.

2. Zitvogel L, Galluzzi L, Smyth MJ and Kroemer G. Mechanism of action of conventional and targeted anticancer therapies: reinstating immunosurveillance. Immunity. 2013; 39(1):74-88.

3. Vacchelli E, Eggermont A, Sautes-Fridman C, Galon J, Zitvogel L, Kroemer G and Galluzzi L. Trial Watch: Tolllike receptor agonists for cancer therapy. Oncoimmunology. 2013; 2(8):e25238.

4. Chen DS and Mellman I. Oncology meets immunology: the cancer-immunity cycle. Immunity. 2013; 39(1):1-10.

5. Zou W and Chen L. Inhibitory B7-family molecules in the tumour microenvironment. Nat Rev Immunol. 2008; 8(6):467-477.

6. Lundin JI and Checkoway H. Endotoxin and cancer. Environ Health Perspect. 2009; 117(9):1344-1350.

7. Karin M. NF-kappaB as a critical link between inflammation and cancer. Cold Spring Harb Perspect Biol. 2009; 1(5):a000141.

8. Sha W, Brune B and Weigert A. The multi-faceted roles of prostaglandin E2 in cancer-infiltrating mononuclear phagocyte biology. Immunobiology. 2012; 217(12):12251232.

9. Jakobsson PJ, Thoren S, Morgenstern R and Samuelsson B. Identification of human prostaglandin E synthase: a microsomal, glutathione-dependent, inducible enzyme, constituting a potential novel drug target. Proc Natl Acad Sci U S A. 1999; 96(13):7220-7225.

10. Bage T, Lindberg J, Lundeberg J, Modeer T and YucelLindberg T. Signal pathways JNK and NF-kappaB, identified by global gene expression profiling, are involved in regulation of TNFalpha-induced mPGES-1 and COX-2 expression in gingival fibroblasts. BMC Genomics. 2010; 11:241.

11. Leclerc P, Idborg H, Spahiu L, Larsson C, Nekhotiaeva N, Wannberg J, Stenberg P, Korotkova M and Jakobsson PJ. Characterization of a human and murine mPGES-1 inhibitor and comparison to mPGES-1 genetic deletion in mouse models of inflammation. Prostaglandins Other Lipid Mediat. 2013; 107:26-34.

12. Kalinski P. Regulation of immune responses by prostaglandin E2. J Immunol. 2012; 188(1):21-28.

13. Wang D and Dubois RN. Eicosanoids and cancer. Nat Rev Cancer. 2010; 10(3):181-193.

14. Obermajer N, Muthuswamy R, Lesnock J, Edwards RP and
Kalinski P. Positive feedback between PGE2 and COX2 redirects the differentiation of human dendritic cells toward stable myeloid-derived suppressor cells. Blood. 2011; 118(20):5498-5505

15. Sinha P, Clements VK, Fulton AM and Ostrand-Rosenberg S. Prostaglandin E2 promotes tumor progression by inducing myeloid-derived suppressor cells. Cancer Res. 2007; 67(9):4507-4513.

16. Monrad SU, Kojima F, Kapoor M, Kuan EL, Sarkar S, Randolph GJ and Crofford LJ. Genetic deletion of mPGES-1 abolishes PGE2 production in murine dendritic cells and alters the cytokine profile, but does not affect maturation or migration. Prostaglandins Leukot Essent Fatty Acids. 2011; 84(3-4):113-121.

17. Feder-Mengus $\mathrm{C}$, Ghosh S, Reschner A, Martin I and Spagnoli GC. New dimensions in tumor immunology: what does 3D culture reveal? Trends Mol Med. 2008; 14(8):333340 .

18. Medina MA, Couturier J, Feske ML, Mahne AE, Turner M, Yu X, Kozinetz CA, Orozco AF, Hutchison AT, Savidge TC, Rodgers JR and Lewis DE. Granzyme B- and Fas ligand-mediated cytotoxic function induced by mitogenic CD28 stimulation of human memory CD4+ T cells. J Leukoc Biol. 2012; 91(5):759-771.

19. Nunes JA, Truneh A, Olive D and Cantrell DA. Signal transduction by CD28 costimulatory receptor on T cells. B7-1 and B7-2 regulation of tyrosine kinase adaptor molecules. J Biol Chem. 1996; 271(3):1591-1598.

20. Lin EY, Jones JG, Li P, Zhu L, Whitney KD, Muller WJ and Pollard JW. Progression to malignancy in the polyoma middle $\mathrm{T}$ oncoprotein mouse breast cancer model provides a reliable model for human diseases. Am J Pathol. 2003; 163(5):2113-2126.

21. Sha W, Olesch C, Hanaka H, Radmark O, Weigert A and Brune B. Necrosis in DU145 prostate cancer spheroids induces COX-2/mPGES-1-derived PGE2 to promote tumor growth and to inhibit $\mathrm{T}$ cell activation. Int J Cancer. 2013; 133(7):1578-1588.

22. Ivascu A and Kubbies M. Rapid generation of single-tumor spheroids for high-throughput cell function and toxicity analysis. J Biomol Screen. 2006; 11(8):922-932.

23. Sekar D, Hahn C, Brune B, Roberts E and Weigert A. Apoptotic tumor cells induce IL-27 release from human DCs to activate Treg cells that express CD69 and attenuate cytotoxicity. Eur J Immunol. 2012; 42(6):1585-1598.

24. Linke B, Pierre S, Coste O, Angioni C, Becker W, Maier TJ, Steinhilber D, Wittpoth C, Geisslinger G and Scholich $\mathrm{K}$. Toponomics analysis of drug-induced changes in arachidonic acid-dependent signaling pathways during spinal nociceptive processing. J Proteome Res. 2009; 8(10):4851-4859.

25. Ley S, Weigert A, Weichand B, Henke N, Mille-Baker $\mathrm{B}$, Janssen RA and Brune B. The role of TRKA signaling in IL-10 production by apoptotic tumor cell-activated 
macrophages. Oncogene. 2013; 32(5):631-640.

26. Fabre JW. The allogeneic response and tumor immunity. Nat Med. 2001; 7(6):649-652.

27. Schreiber RD, Old LJ and Smyth MJ. Cancer immunoediting: integrating immunity's roles in cancer suppression and promotion. Science. 2011; 331(6024):15651570.

28. Franklin RA, Liao W, Sarkar A, Kim MV, Bivona MR, Liu K, Pamer EG and Li MO. The Cellular and Molecular Origin of Tumor-Associated Macrophages. Science. 2014.

29. Laoui D, Van Overmeire E, Movahedi K, Van den Bossche J, Schouppe E, Mommer C, Nikolaou A, Morias Y, De Baetselier $\mathrm{P}$ and Van Ginderachter JA. Mononuclear phagocyte heterogeneity in cancer: different subsets and activation states reaching out at the tumor site. Immunobiology. 2011; 216(11):1192-1202.

30. Mantovani A, Sica A and Locati M. Macrophage polarization comes of age. Immunity. 2005; 23(4):344-346.

31. Treffkorn L, Scheibe R, Maruyama T and Dieter P. PGE2 exerts its effect on the LPS-induced release of TNF-alpha, ET-1, IL-1alpha, IL-6 and IL-10 via the EP2 and EP4 receptor in rat liver macrophages. Prostaglandins Other Lipid Mediat. 2004; 74(1-4):113-123.

32. Brenneis C, Coste O, Altenrath K, Angioni C, Schmidt H, Schuh CD, Zhang DD, Henke M, Weigert A, Brune B, Rubin B, Nusing R, Scholich K and Geisslinger G. Anti-inflammatory role of microsomal prostaglandin $\mathrm{E}$ synthase-1 in a model of neuroinflammation. J Biol Chem. 2011; 286(3):2331-2342.

33. Peters-Golden M. Putting on the brakes: cyclic AMP as a multipronged controller of macrophage function. Sci Signal. 2009; 2(75):pe37.

34. Kubo S, Takahashi HK, Takei M, Iwagaki H, Yoshino T, Tanaka N, Mori S and Nishibori M. E-prostanoid (EP)2/EP4 receptor-dependent maturation of human monocyte-derived dendritic cells and induction of helper T2 polarization. J Pharmacol Exp Ther. 2004; 309(3):1213-1220.

35. Sa-Nunes A, Bafica A, Lucas DA, Conrads TP, Veenstra TD, Andersen JF, Mather TN, Ribeiro JM and Francischetti IM. Prostaglandin E2 is a major inhibitor of dendritic cell maturation and function in Ixodes scapularis saliva. J Immunol. 2007; 179(3):1497-1505.

36. Fabricius D, Neubauer M, Mandel B, Schutz C, Viardot A, Vollmer A, Jahrsdorfer B and Debatin KM. Prostaglandin E2 inhibits IFN-alpha secretion and Th1 costimulation by human plasmacytoid dendritic cells via E-prostanoid 2 and E-prostanoid 4 receptor engagement. J Immunol. 2010; 184(2):677-684.

37. Sharma S, Yang SC, Zhu L, Reckamp K, Gardner B, Baratelli F, Huang M, Batra RK and Dubinett SM. Tumor cyclooxygenase-2/prostaglandin E2-dependent promotion of FOXP3 expression and CD4+ CD25+ T regulatory cell activities in lung cancer. Cancer Res. 2005; 65(12):52115220.
38. Qureshi OS, Zheng Y, Nakamura K, Attridge K, Manzotti C, Schmidt EM, Baker J, Jeffery LE, Kaur S, Briggs Z, Hou TZ, Futter CE, Anderson G, Walker LS and Sansom DM. Trans-endocytosis of CD80 and CD86: a molecular basis for the cell-extrinsic function of CTLA-4. Science. 2011; 332(6029):600-603.

39. Howe LR, Subbaramaiah K, Kent CV, Zhou XK, Chang SH, Hla T, Jakobsson PJ, Hudis CA and Dannenberg AJ. Genetic deletion of microsomal prostaglandin E synthase-1 suppresses mouse mammary tumor growth and angiogenesis. Prostaglandins Other Lipid Mediat. 2013; 106:99-105.

40. Wurz GT, Gutierrez AM, Greenberg BE, Vang DP, Griffey SM, Kao CJ, Wolf $M$ and Degregorio MW. Antitumor effects of L-BLP25 Antigen-Specific tumor immunotherapy in a novel human MUC1 transgenic lung cancer mouse model. J Transl Med. 2013; 11(1):64.

41. Kroemer G, Zitvogel L and Galluzzi L. Victories and deceptions in tumor immunology: Stimuvax. Oncoimmunology. 2013; 2(1):e23687. 